\title{
ASYMPTOTIC NORMALITY OF GENERALIZED L-STATISTICS WITH UNBOUNDED SCORES
}

\author{
R. HELMERS
}

Centre for Mathematics and Computer Science, P.O. Box 4079, 1009 AB Amsterdam, The Netherlands

\section{F.H. RUYMGAART}

Catholic University Nijmegen, 6525 ED Nijmegen, The Netherlands

Received 17 November 1986; revised manuscript received 13 July 1987

Recommended by R.J. Serfling

\begin{abstract}
A central limit theorem for linear combinations of a function of generalized order statistics with unbounded scores is established. The result supplements previous work of Silverman (1983), Serfling (1984) and Akritas (1986) concerning the asymptotic normality of generalized $L$-statistics. Our proof is patterned after the well-known Chernoff-Savage approach. A linear bound for the empirical distribution function of $U$-statistic structure is also derived and subsequently applied in the treatment of certain remainder terms.
\end{abstract}

AMS Subject Classification: Primary 62G05, 62G30; Secondary 62E20.

Key words and phrases: $U$-statistics; empirical processes; $L$-statistics; unbounded scores; asymptotic normality.

\section{Introduction}

For each $n \in N$ let $\xi_{1}, \ldots, \xi_{n}$ be independent and identically distributed (i.i.d) random elements with values in some measurable space $X$ and let, for a fixed but arbitrary $m \in N, \quad h: X^{m} \rightarrow \mathbb{R}$ be a measurable mapping. For each of the $n(m)=n \cdot(n-1) \cdots(n-m+1)$ ordered $m$-tuples $(j(1), \ldots, j(m))$ of $m$ distinct integers taken from $\{1, \ldots, n\}$ we form the random variable (r.v.) $h\left(\xi_{j(1)}, \ldots, \xi_{j(m)}\right)$. Let $X_{1}, \ldots, X_{n(m)}$ be an enumeration of these r.v.'s and note that, although dependent in general, these r.v.'s are still identically distributed with common distribution function (d.f.) $H$, say. It will be assumed throughout that

$H$ is continuous on $\mathbb{R}$,

so that for $i=1, \ldots, n(m)$ the transformed r.v.

$$
H\left(X_{i}\right) \text { has the uniform }(0,1) \text { distribution. }
$$


The empirical d.f. of $H\left(X_{1}\right), \ldots, H\left(X_{n(m)}\right)$ is as usual defined by

$$
\hat{H}_{n(m)}(t)=\frac{1}{n(m)} \sum_{i=1}^{n(m)} 1_{[0, t]}\left(H\left(X_{i}\right)\right), \quad t \in[0,1] .
$$

$\hat{H}_{n(m)}$ is called the empirical df of $U$-statistic structure (cf. Serfling (1984)). Let $X_{1: n(m)} \leq \cdots \leq X_{n(m): n(m)}$ denote the (generalized) order statistics corresponding to $X_{1}, \ldots, X_{n(m)}$. For $J_{n}:[0,1] \rightarrow \mathbb{R}$ with $c_{n(m), i}=J_{n}(i / n(m))$, and measurable $\Psi: \mathbb{R} \rightarrow \mathbb{R}$ with $\Psi_{H}=\Psi\left(H^{-1}\right)$ let us consider the linear combination of the function $\Psi$ applied to the $X_{i: n(m)}$, given by

$$
\begin{aligned}
T_{n} & =\frac{1}{n(m)} \sum_{i=1}^{n(m)} c_{n(m), i} \Psi\left(X_{i: n(m)}\right) \\
& =\int_{0}^{1} J_{n}\left(\hat{H}_{n(m)}(t)\right) \Psi_{H}(t) \mathrm{d} \hat{H}_{n(m)}(t) \text { a.s. }
\end{aligned}
$$

For the special choice $\Psi_{H}=H^{-1}$ this class has been introduced by Serfling (1984) as the class of generalized $L$-statistics, and asymptotic normality has been established in Silverman (1983) and Serfling (1984) for bounded scores $c_{n(m), i}$. A strong law for statistics of the general form (1.4), implying almost sure convergence of $T_{n}$ to its natural limit $\mu(H)=\int_{0}^{1} J(t) \psi_{H}(t) \mathrm{d} t$ for some limiting score function $J$, is contained in Corollary 3.1 of Helmers et al. (1985). We also refer to Janssen et al. (1984) for the asymptotic normality of a general class of statistical functions. Included are, e.g., certain measures of spread as well as generalized $L$-functionals considered by Serfling (1984).

In this note we will prove asymptotic normality for a class of functions $\Psi_{H}$ and for non necessary bounded scores. One of the important tools in the study of unbounded scores is that the empirical d.f. lies, with high probability, above a line through the origin on an interval $[v, 1]$ with $v>0$, so that the random argument of the score function may be replaced by a non-random one. The present Theorem 2 provides a result of this kind without any structural condition on the kernel $h$ and with $v=v_{n}=c(\log n) / n$ for some $c \in(0, \infty)$. The largest possible interval where the above mentioned property may hold true is, of course, obtained for $v=$ $H\left(X_{1: n(m)}\right)$.

Specializing Theorem 2 to the i.i.d. case $m=1$ leads to a result that implies similar statements for the interval $\left[H\left(X_{1: n(1)}\right), 1\right]$, because $H\left(X_{1: n(1)}\right)>v_{n}$ with high probability. For $m=1$, Theorem 2 is good enough to yield the almost optimal order of magnitude $\mathrm{O}\left(n(1)^{\alpha}\right)$ for the scores. For $m>1$, however, the order of magnitude that we obtain is $\mathrm{O}\left(n(m)^{\alpha / m}\right)$. This would again be almost optimal if also for $m>1$, $H\left(X_{1: n(m)}\right)$ the smallest order statistic were bounded below by $v_{n}$ with high probability. It is likely, however, that $H\left(X_{1: n(m)}\right)$ is of smaller order, depending on moment properties of the kernel $h$. The behavior of $X_{1: n(m)}$ seems an interesting open problem. For some information about this point see Aerts et al. (1986).

Our representation in (1.4) is the natural starting point for the Chernoff-Savage 
approach which is also employed in e.g. Moore (1968), Ruymgaart and van Zuijlen (1977) and Beirlant et al. (1982). From a theoretical point of view this method is not so attractive since it doesn't seem to yield theoretically refined results like e.g. the one in Mason (1981). On the other hand the method automatically leads to simple centering constants and the method is of indirect theoretical importance as it hinges on some interesting properties of the empirical d.f. involved. The two properties that we need, one of which seems to be new, are presented in Section 2. In Section 3 we return to the asymptotic normality of the statistics in (1.4).

Results related to our Theorems 2 and 3 were very recently obtained by Akritas (1986). However, rather then considering the empirical d.f. $\hat{H}_{n(m)}$ of $U$-statistic structure and GL-statistics $T_{n}=T_{n}\left(\hat{H}_{n(m)}\right)$ of the form (1.4), Akritas investigated the closely related empirical d.f. $V_{n(m)}$ of von Mises structure (see Serfling (1980), p. 174) and modified GL-statistics $T_{n}=T_{n}\left(V_{n(m)}\right)$. His purpose is to deal with such statistics in the multi-sample case in the presence of random censoring. An important drawback of Akritas results is that he requires, using a different method of proof, a rather restrictive condition on the kernel function $h$ which we are able to avoid. On the other hand, as in our Theorem 3, unbounded scores are permitted. Inequality (A.7) of Theorem A.2 of Akritas (1986), a version of our Theorem 2 for the empirical d.f. $V_{n(m)}$ of von Mises structure, is stated for $v=H\left(X_{1: n(m)}\right)$, under a restrictive regularity condition on the kernel $h$.

\section{Properties of the empirical d.f. of $U$-statistic structure}

Throughout this section we can and will assume without loss of generality that $n=m \cdot v$ for some $v \in \mathbb{N}$, although we will write $n \rightarrow \infty$ rather than $v \rightarrow \infty$. Let $\mathscr{R}(n)$ denote the set of all permutations $(r(1), \ldots, r(n))$ of the numbers $(1, \ldots, n)$ and take $r \in \mathscr{R}(n)$. As in Silverman (1983) we define $\hat{H}_{v, r}$ to be the empirical d.f. of the $v$ r.v.'s. $H\left(h\left(\xi_{r(m j+1)}, \xi_{r(m j+2)}, \ldots, \xi_{r(m j+m)}\right)\right)$ for $j=0, \ldots, v-1$. Note that this subset of $v$ r.v.'s consists of i.i.d. elements so that for the corresponding empirical process

$$
U_{v, r}(t)=v^{1 / 2}\left(\hat{H}_{v, r}(t)-t\right), \quad t \in[0,1],
$$

the usual properties hold true. The empirical process $U_{n}$ of $U$-statistic structure based on all the $X_{1}, \ldots, X_{n(m)}$ is related to the i.i.d. empirical processes in (2.1) according to

$$
U_{n}(t)=n^{1 / 2}\left(\hat{H}_{n(m)}(t)-t\right)=\frac{m^{1 / 2}}{n !} \sum_{r \in \mathscr{R}(n)} U_{v, r}(t), \quad t \in[0,1] .
$$

For arbitrary $\delta \in\left(0, \frac{1}{2}\right)$ let

$$
q_{\delta}(t)=[t(1-t)]^{1 / 2-\delta}, \quad t \in(0,1)
$$

The first property that we need is implied by Silverman (1983, Theorem A) and says that 


$$
\sup _{t \in(0,1)}\left|U_{n}(t)\right| / q_{\delta}(t)=\mathrm{O}_{p}(1) \quad \text { as } n \rightarrow \infty .
$$

In order to prepare for the second property we need the following probability inequality for the i.i.d. empirical processes in (2.1); a proof (for arbitrary dimension) can be found in Einmahl (1987). Let $0 \leq a<b \leq 1$ be arbitrary but fixed and let $a \leq s<t \leq b$. Then we have

$$
P\left(\sup _{a \leq s<t \leq b}-\left(U_{v, r}(t)-U_{v, r}(s)\right) \geq \lambda\right) \leq C \exp \left(\frac{-(1-\varepsilon) \lambda^{2}}{2(b-a)}\right), \quad \lambda \geq 0,
$$

for each $\varepsilon>0$ with $C=C(\varepsilon) \in(0, \infty)$. Note that we consider $-\left(U_{v, r}(t)-U_{v, r}(s)\right)$ rather than $\left|U_{v, r}(t)-U_{v, r}(s)\right|$ since this suffices for our purposes. For this tail the bound appears to be smaller and easier to handle. Using a moment generating function technique to be found in Serfling (1980, Section 5.6), see also Helmers et al. (1985), it will be shown that an analogous inequality holds true for the processes $U_{n}$ in (2.2). Throughout the remainder of this section the symbols $A$ and $C$ will be used as generic constants in $(0, \infty)$ that are independent of all the relevant parameters $(n, \lambda, a$ and $b)$.

Theorem 1. Fix arbitrary $0 \leq a<b \leq 1$. Then we have

$$
P\left(\sup _{a \leq s<t \leq b}-\left(U_{n}(t)-U_{n}(s)\right) \geq \lambda\right) \leq C \exp \left(\frac{-(1-\varepsilon) \lambda^{2}}{2 m(b-a)}\right), \quad \lambda \geq 0 .
$$

Proof. For each $x>0$ the probability in (2.6) is bounded above by

$$
\begin{aligned}
& \exp (-\lambda x) E \exp \left(x \sup _{a \leq s<t \leq b}-\left(U_{n}(t)-U_{n}(s)\right)\right) \\
& \quad \leq \exp (-\lambda x) E \exp \left(\frac{x m^{1 / 2}}{n !} \sum_{x \in \mathbb{R}(n)} \sup _{a \leq s<t \leq b}-\left(U_{v, r}(t)-U_{v, r}(s)\right)\right) \\
& \quad \leq \exp (-\lambda x) \int_{0}^{\infty} P\left(\sup _{a \leq s<t \leq b}-\left(U_{v, r}(t)-U_{v, r}(s)\right) \geq \frac{\log u}{x m^{1 / 2}}\right) \mathrm{d} u \\
& \leq \exp (-\lambda x) C \int_{0}^{\infty} \exp \left(\frac{-(1-\varepsilon)(\log u)^{2}}{x^{2} m(b-a)}\right) \mathrm{d} u \\
& =C \exp (-\lambda x) \int_{-\infty}^{\infty} \exp \left(u-\frac{(1-\varepsilon) u^{2}}{x^{2} m(b-a)}\right) \mathrm{d} u \\
& =C \exp \left(-\lambda x+\frac{x^{2} m(b-a)}{4(1-\varepsilon)}\right)\left(\frac{\pi x^{2} m(b-a)}{1-\varepsilon}\right)^{1 / 2}, \quad \lambda \geq 0 .
\end{aligned}
$$

Minimizing the exponential factor as a function of $x$ and taking into account the square root, we arrive at the exponential bound in (2.6).

Following the method of the proof in Ruymgaart and Wellner (1982, Corollary 
2.4) it is easy to see that with the aid of (2.6) we arrive at the global version

$$
P\left(\sup _{t \geq v} \frac{-U_{n}(t)}{t^{1 / 2}} \geq \lambda\right) \leq C \log (1 / v) \exp \left(-A \lambda^{2}\right), \quad v \in(0,1), \lambda \geq 0 ;
$$

see also Einmahl and Mason (1985, Inequality 1). This inequality entails at once

$$
P\left(\sup _{t \geq v} \frac{-U_{n}(t)}{t} \geq \lambda\right) \leq C \log (1 / v) \exp \left(-A v \lambda^{2}\right), \quad v \in(0,1), \lambda \geq 0 .
$$

We may now formulate the second property of the empirical df of $U$-statistic structure.

Theorem 2. Let us choose $v_{n}=c(\log n) / n$ for some $c \in(0, \infty)$ and $\beta \in(0,1)$ arbitrary but fixed. For any choice of $c \in(0, \infty)$ we have

$$
P\left(\hat{H}_{n(m)}(t) \geq \beta t \forall t \in\left[v_{n}, 1\right]\right) \rightarrow 1 \text { as } n \rightarrow \infty .
$$

For c sufficiently large we even have that $\left\{\hat{H}_{n(m)}(t) \geq \beta\right.$ for all $\left.t \in\left[v_{n}, 1\right]\right\}$ occurs eventually w.p. 1.

Proof. The complement of the event in (2.10) has probability

$$
\begin{aligned}
P\left(\inf _{t \geq v_{n}} \hat{H}_{n(m)}(t) / t \leq \beta\right) & =P\left(\sup _{t \geq v_{n}}\left(t-\hat{H}_{n(m)}(t) / t\right) \geq 1-\beta\right) \\
& =P\left(\sup _{t \geq v_{n}}-U_{n}(t) / t \geq n^{1 / 2}(1-\beta)\right) .
\end{aligned}
$$

Both results now follow immediately from (2.9).

In the i.i.d. case $(m=1)$ relation $(2.10)$ is known to remain true with $v_{n}$ replaced by $H\left(X_{1: n(m)}\right)$; see e.g. Shorack (1972) and, for a.s. results, Shorack and Wellner (1978). Whether this is also the case for arbitrary $m$ is an open question and the answer might depend on the structure of the kernel $h$; see also Aerts et al. (1986). In the i.i.d. case $(m=1)$ almost sure results for non-random $v_{n}$ can be found in Wellner (1978).

\section{Application to GL-statistics}

Let us first formulate sufficient conditions on the functions $J_{n}$ and $\Psi_{H}$ in (1.4). It is convenient to first introduce the linear function

$$
l_{n}(t)=v_{n}+\left(1-2 v_{n}\right) t, \quad t \in[0,1], v_{n}=c(\log n) / n,
$$

for some $c \in(0, \infty)$. The functions $J_{n}$ will be derived from a fixed function $J:(0,1) \rightarrow \mathbb{R}$ according to

$$
J_{n}(t)=J\left(l_{n}(t)\right), \quad t \in[0,1]
$$


It will be assumed that

$$
\left\{\begin{array}{l}
J \text { is continuously differentiable on }(0,1) \text { with } \\
\left|J^{(i)}(t)\right| \leq C[t(1-t)]^{-\alpha-i}, \quad t \in(0,1), \alpha \in(0,1), i \in\{0,1\},
\end{array}\right.
$$

where $J^{(0)}=J$, and that

$$
\left\{\begin{array}{l}
\Psi_{H} \text { is of bounded variation on }(\varepsilon, 1-\varepsilon) \text { for any } \varepsilon>0 \\
\left|\Psi_{H}(t)\right| \leq C[t(1-t)]^{-\beta}, \quad t \in(0,1), \beta \in(0,1)
\end{array}\right.
$$

We finally assume that

$$
\alpha+\beta<\frac{1}{2} .
$$

Theorem 3. Let the conditions described in (3.1)-(3.5) be fulfilled. Then $\sigma^{2}(H)>0$ implies

$$
n^{1 / 2}\left(T_{n}-\mu(H)\right) \stackrel{\mathrm{d}}{\longrightarrow} \mathrm{N}\left(0, \sigma^{2}(H)\right) \text { as } n \rightarrow \infty,
$$

where $T_{n}$ is defined in (1.4), and $\mu(H)=\int_{0}^{1} J(t) \Psi_{H}(t) \mathrm{d} t$,

$$
\sigma^{2}(H)=m^{2} \int_{0}^{1} \int_{0}^{1}(\min (s, t)-s t) J(s) J(t) \mathrm{d} \psi_{H}(t) \mathrm{d} \psi_{H}(s) .
$$

Proof. We may in principle follow the pattern of proof in Beirlant et al. (1982, pp. 427-430) or Ruymgaart and van Zuijlen (1977). Writing

$$
\begin{aligned}
& Z_{0 i}=J_{n}\left(H\left(X_{i}\right)\right) \Psi_{H}\left(H\left(X_{i}\right)\right), \\
& Z_{1 i}=\int_{0}^{1}\left(1_{[0, t]}\left(H\left(X_{i}\right)\right)-t\right) J_{n}^{(1)}(t) \Psi_{H}(t) \mathrm{d} t,
\end{aligned}
$$

we shall first consider

where

$$
A_{n}=\sum_{i=0}^{2} A_{\text {in }}
$$

$$
\begin{aligned}
& A_{0 n}=n^{1 / 2} \frac{1}{n(m)} \sum_{i=1}^{n(m)}\left(Z_{0 i}-E Z_{0 i}\right), \\
& A_{1 n}=n^{1 / 2} \frac{1}{n(m)} \sum_{i=1}^{n(m)} Z_{1 i}
\end{aligned}
$$

and

$$
A_{2 n}=n^{1 / 2}\left(\int_{0}^{1} J_{n}(t) \psi_{H}(t) \mathrm{d} t-\mu(H)\right)
$$

with $\mu(H)$ as in (3.7). Note that $E A_{0 n}=E A_{1 n}=0$ and that $A_{2 n}$ is non-random. Partial integration directly yields that 


$$
A_{0 n}+A_{1 n}=-n^{1 / 2} \frac{1}{n(m)} \sum_{i=1}^{n(m)} \int_{0}^{1}\left(I_{[0, t]}\left(H\left(X_{i}\right)\right)-t\right) J_{n}(t) \mathrm{d} \psi_{H}(t)
$$

a $U$-statistic of degree $m$ with a varying kernel

$$
A^{(n)}\left(\xi_{1}, \ldots, \xi_{m}\right)=-\int_{0}^{1}\left(I_{[0, t]}\left(H\left(h\left(\xi_{1}, \ldots, \xi_{m}\right)\right)\right)-t\right) J_{n}(t) \mathrm{d} \psi_{H}(t)
$$

depending on $n$. Let $U_{n}$ denote the $U$-statistic of degree $m$, with fixed kernel, which is obtained from (3.14) by replacing $J_{n}$ by $J$.

To establish the asymptotic normality of $A_{n}$ (cf. (3.10)) we first note that the central limit theorem for $U$-statistics (see Serfling (1980), p. 192) directly yields that

$$
U_{n} \stackrel{\mathrm{d}}{\longrightarrow} \mathrm{N}\left(0, \sigma^{2}(H)\right) \text { as } n \rightarrow \infty,
$$

with $\sigma^{2}(H)$ as in (3.7). In addition we shall prove that

$$
A_{0 n}+A_{1 n}-U_{n} \stackrel{\mathrm{p}}{\longrightarrow} 0 \text { as } n \rightarrow \infty
$$

and also that

$$
A_{2 n} \rightarrow 0 \text { as } n \rightarrow \infty
$$

Together (3.16)-(3.18), combined with (3.10), gives the desired result: $A_{n} \stackrel{\mathrm{d}}{\longrightarrow}$ $\mathrm{N}\left(0, \sigma^{2}(H)\right)$ as $n \rightarrow \infty$. To verify (3.17) we first apply Chebychev's inequality and the elementary inequality $\sigma^{2}(X+Y) \leq 2 \sigma^{2}(X)+2 \sigma^{2}(Y)$ to find that it suffices clearly to show that both

and

$$
\sigma^{2}\left(n^{1 / 2} n(m)^{-1} \sum_{i=1}^{n(m)}\left(J_{n}-J\right)\left(H\left(X_{i}\right)\right) \psi_{H}\left(H\left(X_{i}\right)\right)\right)
$$

$$
\sigma^{2}\left(n^{1 / 2} n(m)^{-1} \sum_{i=1}^{n(m)} \int_{0}^{1}\left(I_{[0, t]}\left(H\left(X_{i}\right)\right)-t\right)\left(J_{n}^{(1)}(t)-J^{(1)}(t)\right) \psi_{H}(t) \mathrm{d} t\right)
$$

tend to zero, as $n \rightarrow \infty$. With the aid of Lemma A on page 183 of Serfling (1980) we easily check that the variances in (3.19) and (3.20) are respectively of the order

and

$$
\mathrm{O}\left(\int_{0}^{1}\left(\left(J_{n}-J\right)(s) \psi_{H}(s)\right)^{2} \mathrm{~d} s\right)
$$

$$
\mathrm{O}\left(\int_{0}^{1} \int_{0}^{1}(\min (s, t)-s t)\left|J_{n}^{(1)}(s)-J^{(1)}(s)\right|\left|J_{n}^{(1)}(t)-J^{(1)}(t)\right|\left|\psi_{H}(t)\right|\left|\psi_{H}(s)\right| \mathrm{d} s \mathrm{~d} t\right)
$$

as $n \rightarrow \infty$. In view of the assumptions (3.1)-(3.5) one directly verifies that the integrals appearing in (3.21) and (3.22) both tend to zero as $n \rightarrow \infty$, and (3.17) follows.

It remains to check (3.18). The same argument involving the assumptions (3.1)-(3.5) also yields that 


$$
n^{1 / 2} \int_{0}^{1}\left(J_{n}(t)-J(t)\right) \psi_{H}(t) \mathrm{d} t \rightarrow 0 \quad \text { as } n \rightarrow \infty .
$$

Thus (3.18) indeed holds and the asymptotic normality of $A_{n}$ is proved. It remains to show that

$$
\begin{aligned}
B_{n} & =n^{1 / 2}\left(T_{n}-\mu(H)\right)-A_{n} \\
& =n^{1 / 2} \int_{0}^{1}\left\{J_{n}\left(\hat{H}_{n(m)}(t)\right)-J_{n}(t)\right\} \Psi_{H}(t) \mathrm{d} \hat{H}_{n(m)}-A_{1 n} \\
& \stackrel{\mathrm{p}}{\longrightarrow} 0 \text { as } n \rightarrow \infty .
\end{aligned}
$$

Let us briefly write

$$
B_{n}=n^{1 / 2} \int_{0}^{1}(\cdots) \mathrm{d} \hat{H}_{n(m)}-n^{1 / 2} \int_{0}^{1}(\cdots) \mathrm{d} t .
$$

To prove (3.23) is suffices to prove that each of the integrals

$$
\begin{array}{ll}
B_{1 n}=n^{1 / 2} \int_{0}^{v}(\cdots) \mathrm{d} \hat{H}_{n(m)}, & B_{2 n}=n^{1 / 2} \int_{1-\nu}^{1}(\cdots) \mathrm{d} \hat{H}_{n(m)}, \\
B_{3 n}=n^{1 / 2} \int_{0}^{v}(\cdots) \mathrm{d} t, & B_{4 n}=n^{1 / 2} \int_{1-\nu}^{1}(\cdots) \mathrm{d} t
\end{array}
$$

converges to 0 in probability as both $v \downarrow 0$ and $n \rightarrow \infty$, along with

$$
n^{1 / 2} \int_{\nu}^{1-\nu}(\cdots) \mathrm{d} \hat{H}_{n(m)}-n^{1 / 2} \int_{\nu}^{1-v}(\cdots) \mathrm{d} t \stackrel{\mathrm{p}}{\longrightarrow} 0 \text { as } n \rightarrow \infty,
$$

for each $v \in\left(0, \frac{1}{2}\right)$.

In order to illustrate the use of the properties (2.4) and (2.10) let us by way of an example consider $B_{3 n}$. For any $v \in(0,1)$ we apply the mean value theorem to the factor within the brackets of the integral and find that

$$
J_{n}\left(\hat{H}_{n(m)}(t)\right)-J_{n}(t)=\left(\hat{H}_{n(m)}(t)-t\right)\left(1-2 v_{n}\right) J^{(1)}\left(l_{n}\left(t_{n}\right)\right),
$$

where $t_{n}$ is a random point between $t$ and $\hat{H}_{n(m)}(t)$ and where $l_{n}$ is defined in (3.1). By assumption (3.3) it is clear that

$$
\left|J^{(1)}\left(l_{n}\left(t_{n}\right)\right)\right| \leq C\left[v_{n}\left(1-v_{n}\right)\right]^{-\alpha-1} \leq C[t(1-t)]^{-\alpha-1} \text { for } 0<t \leq v_{n} .
$$

Using $C$ as a generic constant, the same assumption jointly with Theorem 2 yields that, for arbitrary fixed $\beta \in(0,1)$,

$$
\left|J^{(1)}\left(l_{n}\left(t_{n}\right)\right)\right| \leq C[\beta t(1-\beta t)]^{-\alpha-1} \leq C[t(1-t)]^{-\alpha-1} \text { for } v_{n} \leq t \leq v,
$$

with arbitrarily high probability for $n$ sufficiently large. Property (2.4) entails that, with $C$ generic again,

$$
n^{1 / 2}\left(\hat{H}_{n(m)}(t)-t\right) \leq C[t(1-t)]^{1 / 2-\delta} \text { for } t \in(0,1) \text { and } n \in \mathbb{N},
$$

with arbitrarily high probability. 
Combining (3.28)-(3.31) and using assumption (3.4) it follows that

$$
\begin{aligned}
\left|B_{3 n}\right| & \leq C \int_{0}^{\nu}[t(1-t)]^{-\alpha-1}[t(1-t)]^{-\beta} \mathrm{d} t \\
& =C \int_{0}^{\nu}[t(1-t)]^{-1 / 2-\alpha-\beta-\delta} \mathrm{d} t,
\end{aligned}
$$

with arbitrarily high probability for $n$ sufficiently large.

This last integral decreases to 0 as $v \downarrow 0$ provided that we choose $\delta$ so as to satisfy $0<\delta<\frac{1}{2}-\alpha-\beta$; this can be done because of assumption (3.5). This proves that $B_{3 n}$ converges to 0 in probability as $n \rightarrow \infty$ and $v \downarrow 0$.

\section{An example}

In this section we discuss very briefly an specific example of an GL-statistic with unbounded scores.

Let us take $X=\mathbb{R}, m=2$ and

$$
h\left(\xi_{1}, \xi_{2}\right)=\left|\xi_{1}-\xi_{2}\right|
$$

and let the common distribution function $F$ of the $\xi_{i}$ 's be given by

$$
F(x)=\Phi(x / \tau), \quad-\infty<x<\infty,
$$

the normal distribution function with mean zero and variance $\tau^{2}$.

Consider the GL-statistic $T_{n}$ (cf. (1.4)) with scores $C_{i n(2)}$ satisfying (3.2), where

$$
J(t)=2^{-1 / 2} \Phi^{-1}\left(\frac{1+t}{2}\right), \quad 0<t<1 .
$$

Application of Theorem 3 directly yields

where

$$
n^{1 / 2}\left(T_{n}-\mu(H)\right) \stackrel{\mathrm{d}}{\longrightarrow} \mathrm{N}\left(0, \sigma^{2}(H)\right)
$$

$$
\begin{aligned}
& H(x)=2 \Phi\left(\frac{x}{\sqrt{2} \tau}\right)-1, \quad-\infty<x<\infty, \\
& \mu(H)=\int_{0}^{1} J(t) H^{-1}(t) \mathrm{d} t=\tau,
\end{aligned}
$$

and

$$
\begin{aligned}
\sigma^{2}(H) & =4 \tau^{2} \int_{0}^{1} \int_{0}^{1}(s \wedge t-s t) \Phi^{-1}\left(\frac{1+s}{2}\right) \Phi^{-1}\left(\frac{1+t}{2}\right) \mathrm{d} \Phi^{-1}\left(\frac{1+s}{2}\right) \mathrm{d} \Phi^{-1}\left(\frac{1+t}{2}\right) \\
& =2 \tau^{2}
\end{aligned}
$$

where we have used the formula displayed at the top of page 128 of Albers et al. (1976) to compute the double integral in (4.7). It follows from (4.4), (4.6) and (4.7) that 


$$
n^{1 / 2}\left(T_{n}-\tau\right) \stackrel{\mathrm{d}}{\longrightarrow} \mathrm{N}\left(0,2 \tau^{2}\right) .
$$

Clearly $T_{n}$ is a weakly consistent and asymptotically normally distributed, estimator of normal scale $\tau$. Because the Cramér-Rao bound in the normal scale problem equals $2^{-1} \tau^{2}$, we can conclude from (4.8) that $T_{n}$ has asymptotic efficiency 0.25 . Also note that it is easily inferred from Corollary 3.1 of Helmers et al. (1985) that $T_{n}$ is not only weakly consistent, but strongly consistent as well in estimating $\tau$.

\section{Acknowledgement}

The authors wish to thank John Einmahl and David Mason for some useful comments.

\section{References}

Aerts, M., P. Janssen and D.M. Mason (1986). Glivenko-Cantelli convergence for weighted empirical and quantile processes of $U$-statistic structure. In: Yu. V. Prohorov et al., Eds., Proc. IVth Vilnius Conference, Probab. Theory and Math. Statist., Vol. 1. VNU Science Press, Haarlem, 23-30.

Albers, W., P.J. Bickel and W.R. van Zwet (1976). Asymptotic expansions for the power of distribution free tests in the one-sample problem. Ann. Statist. 4 (1), 108-156.

M.G. Akritras (1986). Empirical processes associated with $V$-statistics and a class of estimators under random censoring. Ann. Statist. 14 (2), 619-637.

Beirlant, J., E.C. van der Meulen, F.H. Ruymgaart and M.C.A. van Zuijlen. (1982). On functions bounding the empirical distribution of uniform spacings. Z. Wahrsch. Verw. Gebiete 61, 417-430.

Einmahl, J.H.J. (1987), Multivariate Empirical Processes. CWI Tract 32, Amsterdam.

Einmahl, J.H.J. and D.M. Mason (1985). Bounds for weighted multivariate empirical distribution functions. Z. Wahrsch. Verw. Gebiete 70, 563-571.

Helmers, R., P. Janssen and R.J. Serfling (1985). Glivenko-Cantelli properties of some generalized empirical df's and strong convergence of generalized $L$-statistics. Technical Report No. 460, Department of Mathematical Sciences, The Johns Hopkins University. Probab. Theory and Related Fields, to appear.

Janssen, P., R.J. Serfling and N. Veraverbeke, (1984). Asymptotic normality for a general class of statistical functions and applications of measures of spread. Ann. Statist. 12, 1369-1379.

Mason, D.M. (1981). Asymptotic normality of linear combinations of order statistics with a smooth score function. Ann. Statist. 9, 899-908.

Moore, D.S. (1968). An elementary proof of asymptotic normality of linear functions of order statistics. Ann. Math. Statist. 39, 263-265.

Ruymgaart, F.H. and J.A. Wellner, (1984). Some properties of weighted multivariate empirical processes. Statist. Decisions 2, 199-223.

Ruymgaart, F.H. and M.C.A. van Zuylen, (1977). Asymptotic normality of linear combinations of order statistics in the non-i.i.d. case. Proc. Koninklijke Nederl. Akad. Wetensch. Ser. A 80 (5), 432-447.

Serfling, R.J. (1980). Approximation Theorems of Mathematical Statistics. Wiley, New York.

Serfling, R.J. (1984). Generalized L-, $M$ - and R-statistics. Ann. Statist. 12 (1), 76-86.

Shorack, G.R. (1972). Functions of order statistics. Ann. Math. Statist. 43, 412-427. 
Shorack, G.R. and J.A. Wellner (1978). Linear bounds on the empirical distribution function. Ann. Probab. 6, 349-353.

Silverman, B.W. (1983). Convergence of a class of empirical distribution functions of dependent random variables. Ann. Probab. 11, 745-751.

Wellner, J.A. (1978). Limit theorems for the ratio of the empirical distribution function to the true distribution function. Z. Wahrsch. Verw. Gebiete 45, 73-88. 